\title{
MIRAS End-to-End Calibration: Application to SMOS L1 Processor
}

\author{
Ignasi Corbella, Member, IEEE, Francesc Torres, Member, IEEE, Adriano Camps, Senior Member, IEEE, \\ Andreas Colliander, Manuel Martín-Neira, Member, IEEE, Serni Ribó, Kimmo Rautiainen, \\ Nuria Duffo, Member, IEEE, and Mercè Vall-llossera, Member, IEEE
}

\begin{abstract}
End-to-end calibration of the Microwave Imaging Radiometer by Aperture Synthesis (MIRAS) radiometer refers to processing the measured raw data up to dual-polarization brightness temperature maps over the earth's surface, which is the level 1 product of the Soil Moisture and Ocean Salinity (SMOS) mission. The process starts with a self-correction of comparators offset and quadrature error and is followed by the calibration procedure itself. This one is based on periodically injecting correlated and uncorrelated noise to all receivers in order to measure their relevant parameters, which are then used to correct the raw data. This can deal with most of the errors associated with the receivers but does not correct for antenna errors, which must be included in the image reconstruction algorithm. Relative S-parameters of the noise injection network and of the input switch are needed as additional data, whereas the whole process is independent of the exact value of the noise source power and of the distribution network physical temperature. On the other hand, the approach relies on having at least one very well-calibrated reference receiver, which is implemented as a noise injection radiometer. The result is the calibrated visibility function, which is inverted by the image reconstruction algorithm to get the brightness temperature as a function of the director cosines at the antenna reference plane. The final step is a coordinate rotation to obtain the horizontal and vertical brightness temperature maps over the earth. The procedures presented are validated using a complete SMOS simulator previously developed by the authors.
\end{abstract}

Index Terms-Calibration, data processing, image reconstruction, microwave radiometry, synthetic aperture imaging.

\section{INTRODUCTION}

$\mathbf{T}$ HE Soil Moisture and Ocean Salinity (SMOS) mission is the second selected opportunity mission on the Earth Observation Program of the European Space Agency (ESA) [1]. Over the ocean, its objective is to produce sea surface salinity global maps with $200-\mathrm{km}$ spatial resolution and accuracy of 0.1 psu every 10-30 days. Over land, it will provide soil moisture global maps at $0.04 \mathrm{~m}^{3} / \mathrm{m}^{3}$ accuracy and vegetation water

Manuscript received April 20, 2004; revised October 7, 2004. This work was supported in part by the European Aeronautic Defence and Space Company Casa espacio and in part by the European Space Agency as part of the SMOS PLM Phase B Contract (16769/02/NL/GS) and in part by the Spanish Administration under Grant TIC2002-04451-C02-01.

I. Corbella, F. Torres, A. Camps, N. Duffo, and M. Vall-llossera are with the Department of Signal Theory and Communications. Universitat Politècnica de Catalunya, 08034 Barcelona, Spain (e-mail: corbella@tsc.upc.es).

A. Colliander and K. Rautiainen are with the Laboratory of Space Technology, Helsinki University of Technology, FIN-02015 HUT, Finland (e-mail: andreas.colliander@hut.fi).

M. Martín-Neira is with the European Space Agency (ESA-ESTEC), 2200 Noordwijk, The Netherlands (e-mail: Manuel.Martin-Neira@esa.int).

S. Ribó is with the Institut d'Estudis Espacials de Catalunya, 08034 Barcelona, Spain (e-mail: ribo@ieec.fcr.es).

Digital Object Identifier 10.1109/TGRS.2004.840458

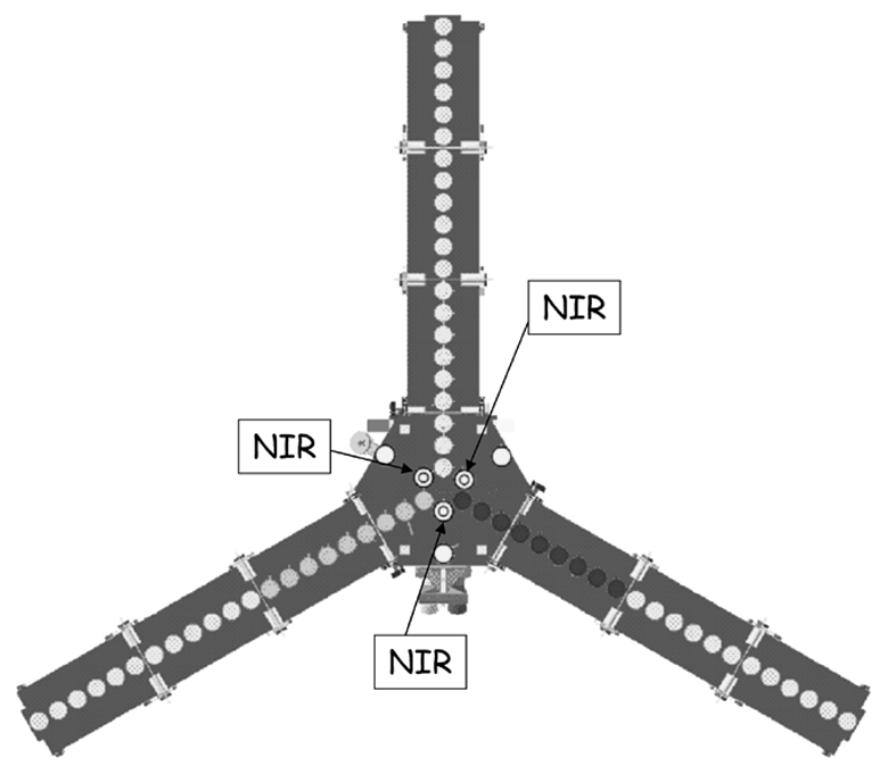

Fig. 1. Mechanical configuration of MIRAS. Courtesy of EADS-CASA espacio.

content at $0.2 \mathrm{~kg} / \mathrm{m}^{2}$ every three days with a spatial resolution of $50 \mathrm{~km}$. Finally, over the cryosphere it will contribute to the improvement of the monitoring of snow layer and multilayer structure of ice. The mission is currently in its phase $\mathrm{C}$, and it is scheduled to be launched in early 2007.

The single payload of the mission is the Microwave Imaging Radiometer by Aperture Synthesis (MIRAS) [2]. It will provide brightness temperature images at L-band after level 1 processing of the raw data. This radiometer will be the first spaceborne instrument to use interferometric aperture synthesis, a technique suggested back in the 1980's as an alternative to real aperture radiometry for earth observation [3]. The whole instrument includes 69 L-band state-of-the-art high-sensitivity receivers, three of them capable to operate as accurate, highly stable noise injection radiometers (NIR). Each receiver has a wide beamwidth antenna and can operate alternatively in two orthogonal polarizations, except the NIRs that are fully polarimetric. The antennas are evenly distributed along a "Y-shape" structure (Fig. 1) providing a spatial resolution similar to that of a real aperture antenna having the same overall dimension as the whole instrument.

This paper presents a description of the processing needed to pass from the MIRAS level 0 data to the level 1 product, this one consisting of horizontal and vertical brightness temperatures on the earth's surface. The second section gives a description of the different processing levels, while the remaining 
ones are devoted to giving an insight on the different processing steps. These are grouped into two main procedures named calibration and inversion. For the first one a new approach based on two-level noise injection is presented and formulas are given. For the second one, a comprehensive description of the required processing is done while specific algorithms are properly referenced to open literature.

\section{DATA LEVELS ZERO AND ONE}

A general overview of the SMOS data levels can be found in the mission web site at http://www.esa.int/export/esaLP/ smos.html. Although the specific products that each level will contain are still being discussed in detail at the date of writing this paper, the main product definitions are already established and are summarized below.

Level zero is basically the instrument's output received by the ground station. Apart from housekeeping and ancillary data, such as readouts of thermal sensors at selected locations on the instrument and UTC time, it includes the following (see Section III):

- digital correlator counts;

- Power Measurement System (PMS) output voltages;

- NIR outputs.

Scientific SMOS level 1A products are the so-called "Calibrated Visibilities" that will be properly defined in Section IV. Within each level 1A product, vectors of calibrated visibilities are arranged as snapshots, i.e., referred to a single integration time. Level $1 \mathrm{~A}$ products are physically consolidated in pole-topole time-based segments (i.e., using time-based segments instead of geographic segments). These time-based segments are thus related to the location of the spacecraft rather than the location of the observation data.

The level 1B products are the output of the image reconstruction of the SMOS observation measurements and consist of geolocated vectors of brightness temperatures in the antenna polarization reference frame.

Level 1C are geolocated vectors of brightness temperatures on the ground pixel polarization reference frame. Faraday rotation must be taken into account, and a land-water mask must be used in order to have the two areas computed separately. The input data needed for this level are the following:

- satellite orbit and attitude;

- data needed to correct the Faraday rotation;

- land/sea mask to separately process brightness temperatures.

Level $1 \mathrm{C}$ products are geographically sorted as a continuous strip and constitute the necessary input to level 2 processing.

The products defined so far must be complemented with ancillary data, assumed to be available from on-ground measurements performed prior to the instrument launching. In particular, antenna ohmic losses and relative S-parameters of the noise distribution network are needed for level 1A computation. Also, receiver nonlinearity and cross-coupling of noise injection between channels is needed as auxiliary data for the NIR.

\section{INSTRUMENT RAW DATA}

The mechanical layout of MIRAS consists of a hexagonal hub and three arms forming a Y-shape (Fig. 1). The hub has 12 stan-

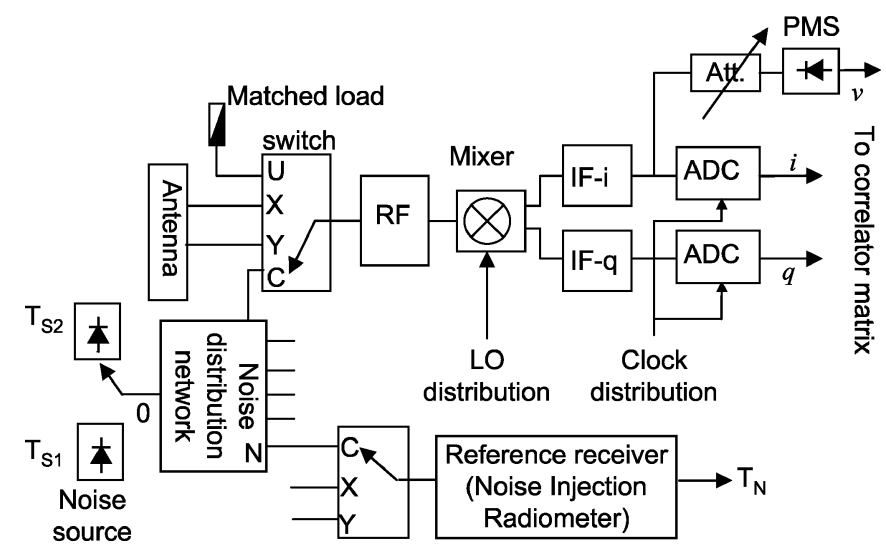

Fig. 2. Schematic block diagram of a receiver.

dard receivers and three NIRs. To allow in-orbit deployment, each arm has three segments with six antennas each, so that the total number of receivers in the hub and the arms is 69. Fig. 2 shows a simplified block diagram of a given receiver along with part of the calibration hardware. The four-way input switch selects one of two antenna outputs (X or Y), uncorrelated noise from a resistor (U), or correlated noise from the noise distribution network $(\mathrm{C})$. The radio-frequency $(\mathrm{RF})$ part amplifies and filters the input signal to 1404-1423 MHz. The mixer shifts this band to an intermediate frequency at 8-27 $\mathrm{MHz}$ using a local oscillator common to all receivers. Two intermediate frequency (IF) output signals are produced, namely the in-phase $(i)$ and quadrature $(q)$ components. One of them is sent to the PMS consisting of a diode detector and an integrator providing a low-frequency voltage proportional to the system noise temperature, which includes the antenna temperature and the receiver noise temperature. Simultaneously, both IF outputs are clipped using zero-voltage comparators to get digital signals which are in turn sent to a centralized matrix of one-bit two-level correlators. In addition to the receivers' output signals, fixed values (digital 1 and 0 ) are also present in the correlator matrix. In this way, the following five different correlation products are available:

- between $i$ channels of different receivers;

- between $q$ and $i$ channels of different receivers;

- between $q$ and $i$ channels of same receivers;

- between " 0 " and $i$ and $q$ channels;

- between " 1 " and $i$ and $q$ channels.

Each individual cell in the correlator matrix is an exclusive NOR gate, so its output is 1 only if the two inputs are identical. The digital correlation is measured by accumulating the output during the integration time at a rate given by the clock frequency $f_{s}=55.84 \mathrm{MHz}$. At the end of the integration time, the counts are read and the accumulator reset for the following period. The correlator output is thus the absolute number of times (counts) that the two input signals coincide during the integration time.

The NIR consists of two independent receivers connected to a dual-polarization antenna and to an internal noise injection circuitry, so that all four Stokes parameters can be measured using the correlator matrix [4]. Its operation is based on an internal noise source which is used to balance the input signal to the receivers. Due to this, the effect of the receiver gain and offset variations are removed and thus the inherent stability of a noise injection radiometer is high [5]. The NIR is designed to measure the fully polarimetric antenna temperature and also, with the ap- 
propriate selection of a switch, the power coming from the noise distribution network (NDN) (Fig. 2). The specific NIR raw data are the noise injection pulse length and the physical temperature of the receivers.

The correlator counts, all PMS voltages and NIR outputs are the raw data sent to ground by MIRAS.

\section{VisiBILITY FUNCTION}

To compute the visibility function, the correlator counts are first preprocessed to eliminate comparators offsets and quadrature errors. Actual calibration is performed afterward by injecting correlated and uncorrelated noise at receivers' inputs. This is used to estimate the system temperatures needed to denormalize the visibilities and also to correct the in-phase and amplitude errors in the correlations due to receivers different frequency responses. The procedure is based on using the NIRs as reference receivers, so the stability of the calibration will ultimately depend on the stability of these radiometers. In orbit the variations of the physical temperature of the NIR are in order of some degrees. Although the NIR is inherently stable, the effect of these changes is significant, and software correction must be applied in the data processing. Required parameters for the correction are solved on-ground. With a prototype of the NIR, it has been demonstrated that the adequate stability can be achieved with this kind of method.

The process cannot deal with antenna imperfections, since the noise is injected through a switch located after the antenna. Therefore, antenna pattern errors must be on-ground characterized and taken into account in the image reconstruction process.

\section{A. Correlator Offset and Quadrature Corrections}

The correlator counts $N_{c}$ are first converted to digital correlation using $Z=2 N_{c} / N_{c_{\max }}-1$, where $N_{c_{\max }}$ is the number of counts obtained when two identical signals are applied to the correlator cell inputs. The digital correlation $Z$ is a real number ranging from -1 to +1 . Theoretically, the normalized correlation of the corresponding analog Gaussian signals is related to this digital correlation by [6]

$$
\mu=\sin \left(\frac{\pi}{2} Z\right)
$$

which is only valid for comparators having zero offset. If this offset is small enough, it can be corrected if $\mu$ is computed by solving the following nonlinear equation [7] in which (1) is used as initial solution:

$$
Z=\frac{2}{\pi} \arcsin \mu-\frac{2}{\sqrt{1-\mu^{2}}}\left(\mu X_{01}^{2}+\mu Y_{01}^{2}-2 X_{01} Y_{01}\right)
$$

where for one of the two input signals $X_{01}=1 / 4\left(Z_{0}-Z_{1}\right)$, in which $Z_{0}$ and $Z_{1}$ are the digital correlations of the input signal with all ones and all zeroes, respectively. The same applies to $Y_{01}$ for the other signal.

Cross-correlations are measured for a large number of signal pairs corresponding to the in-phase and quadrature outputs of the receivers. After performing the processing described so far, the following real normalized correlations are obtained: $\mu_{k j}^{i i}$, $\mu_{k j}^{q i}$ and $\mu_{k k}^{q i}$ where $k$ and $j(k<j)$ are the receiver numbers and $i$ or $q$ denotes the in-phase or quadrature channel of the corresponding receiver. Note that the first two correlations apply to different receivers, while the last one to the $i$ and $q$ outputs of a the same receiver. Denoting by $b_{k}^{i}(t)$ and $b_{k}^{q}(t)$ the analytic signals of the two outputs of receiver $k$, taking into account the phase shift introduced by the mixer in the $q$ branch, any of the above normalized correlations can be written as [8]

$$
\mu_{k j}^{\alpha \beta}=\frac{\Re e\left[e^{j \Delta \phi_{\mathrm{LO}}}\left\langle b_{k}^{\alpha}(t) b_{j}^{\beta}(t)^{*}\right\rangle\right]}{\sqrt{\left\langle\left|b_{k}^{\alpha}(t)\right|^{2}\right\rangle\left\langle\left|b_{j}^{\beta}(t)\right|^{2}\right\rangle}}
$$

where $\alpha$ and $\beta$ should be replaced by $i$ or $q$ depending on the receiver output being considered and $\Delta \phi_{\mathrm{LO}}$ accounts for the local oscillator nominal phase difference between the in-phase and quadrature outputs: $\Delta \phi_{\mathrm{LO}}=0$ if $\alpha=\beta$ and $\Delta \phi_{\mathrm{LO}}=$ $-\pi / 2$ if $\alpha \beta=q i$. Now, using the results of [9], (3) can be expressed as

$$
\mu_{k j}^{\alpha \beta}=\frac{\Re e\left[e^{j \Delta \phi_{\mathrm{LO}}} \tilde{r}_{k j}^{\alpha \beta}(0) V_{k j}\right]}{\sqrt{T_{\mathrm{sys}_{k}} T_{\text {sys }_{j}}}}
$$

where the system temperatures in the denominator are assumed equal for both IF branches and are given by $T_{\mathrm{sys}_{k}}=T_{a_{k}}+T_{R_{k}}$ in which $T_{a_{k}}$ is the antenna temperature and $T_{R_{k}}$ the equivalent noise temperature of receiver $k$. The fringe washing function at zero lag $\tilde{r}_{k j}^{\alpha \beta}(0)$ is related to the frequency domain complex transfer functions of the corresponding channels $H(f)$ by

$$
\tilde{r}_{k j}^{\alpha \beta}(0)=\frac{1}{\sqrt{B_{k}^{\alpha} B_{j}^{\beta}} \sqrt{G_{k}^{\alpha} G_{j}^{\beta}}} \int_{0}^{\infty} H_{k}^{\alpha}(f) H_{j}^{\beta}(f)^{*} d f
$$

where $G$ stands for the power gain and $B$ for noise-equivalent bandwidth. Finally, $V_{k j}$ in (4) is the so-called calibrated visibility, the level 1A product of SMOS and directly related to the brightness temperature of the scene by a Fourier-like integral transform [see (22) in Section VI].

It should be noted that $\tilde{r}_{k j}^{\alpha \beta}(0)$ carries the information of the in-phase and quadrature errors, as well as the nonseparable amplitude and phase errors. Given two receivers $k$ and $j$ the fringe washing functions of the (ii) and (qi) signals can be written approximately as [8]

$$
\tilde{r}_{k j}^{i i}(0)=G_{k j} e^{-\frac{j\left(\theta_{q j}-\theta_{q k}\right)}{2}} \quad \tilde{r}_{k j}^{q i}(0)=G_{k j} e^{-\frac{j\left(\theta_{q j}+\theta_{q k}\right)}{2}}
$$

where $G_{k j}$ is the common part of the fringe washing function to both $i$ and $q$ branches and $\theta_{q k}$ is the quadrature error of receiver $k$.

For the special case of $k=j$ and $\alpha \beta=q i$,(4) and (6) reduce to

$$
\begin{aligned}
\mu_{k k}^{q i} & =\Re e\left[e^{-\frac{j \pi}{2}} \tilde{r}_{k k}^{q i}(0)\right] \\
& =\Im m\left[\tilde{r}_{k k}^{q i}(0)\right] \\
\tilde{r}_{k k}^{q i}(0) & =e^{-j \theta_{q k}}
\end{aligned}
$$

from which the quadrature error of any receiver is easily retrieved

$$
\theta_{q k}=-\arcsin \left(\mu_{k k}^{q i}\right)
$$


(4)

Once the quadrature error is known, $V_{k j}$ comes after solving

$$
V_{k j}=\frac{\sqrt{T_{\mathrm{sys}_{k}} T_{\mathrm{sys}_{j}}}}{G_{k j}} M_{k j}
$$

where $M_{k j}$ is the so-called quadrature-corrected normalized correlation

$$
M_{k j}=\frac{1}{\cos \theta_{q k}}\left(\Re e\left[M_{1} \mu_{k j}\right]+j \Im m\left[M_{2} \mu_{k j}\right]\right)
$$

where $\mu_{k j}=\mu_{k j}^{i i}+j \mu_{k j}^{q i}$ is the normalized complex correlation and $M_{1}$ and $M_{2}$ depend exclusively on the quadrature error of receivers $k$ and $j$

$$
M_{1}=\cos \Theta_{k j}^{\prime}+j \sin \Theta_{k j} \quad M_{2}=\cos \Theta_{k j}+j \sin \Theta_{k j}^{\prime}
$$

where

$$
\Theta_{k j}=\frac{\theta_{q j}}{2}-\frac{\theta_{q k}}{2} \quad \Theta_{k j}^{\prime}=\frac{\theta_{q j}}{2}+\frac{\theta_{q k}}{2}
$$

and the quadrature error is given by (9). The quadrature corrected normalized correlation $M_{k j}$ is continuously computed from the measurements $\mu_{k j}^{i i}, \mu_{k j}^{q i}$, and $\mu_{k k}^{q i}$.

According to (10), to get the calibrated visibility the system temperatures and the fringe-washing factor $G_{k j}$ must be measured. This is done by periodically injecting noise to the receivers' inputs and measuring both the total power and the quadrature corrected normalized correlation.

\section{B. System Temperatures}

System temperatures are measured using the PMS available in all receivers. In order to calibrate them, two different levels of noise are sequentially injected. For one of such levels, the PMS reading is

$$
v_{k}=v_{\mathrm{off}_{k}}+G_{k} T_{\mathrm{sys}_{k}}^{C}
$$

where $v_{\text {off }}$ is the detector offset, $G_{k}$ the power gain, and $T_{\text {sys }_{k}}^{C}$ the total system temperature referred to the switch " $\mathrm{C}$ " input plane

$$
T_{\text {sys }_{k}}^{C}=T_{S}\left|S_{k 0}\right|^{2}+T_{n}+T_{R k}
$$

where $T_{R k}$ is the receiver noise temperature, $T_{n}$ the noise distribution network contribution, $T_{S}$ the source noise temperature, and $S_{k 0}$ the $S$-parameter of the distribution network from the input to receiver $k$ (the source port is numbered as 0 ). The contributions of $T_{n}$ and $T_{R k}$ are cancelled out by injecting two levels of correlated noise and subtracting the resulting equations (14) [10].

The next step consists of referring the system temperatures of all receivers to the NIR measurements, since this is used as a well-calibrated reference receiver. By combining (14) and (15), the system temperature of any receiver can be written as

$$
T_{\mathrm{sys}_{k}}^{C}=\frac{v_{k}-v_{\mathrm{fff}_{k}}}{v_{k}(2)-v_{k}(1)} \frac{\left|S_{k 0}\right|^{2}}{\left|S_{N 0}\right|^{2}}\left[T_{\mathrm{sys}_{N}}^{C}(2)-T_{\mathrm{sys}_{N}}^{C}(1)\right]
$$

where the subscript $N$ refers to the port number of the noise distribution network to which the NIR is connected (Fig. 2), and (1) and (2) refer to both levels of correlated noise. Since the NIR is independently calibrated using an internal load and cold space views (see Section VI), the term $T_{\mathrm{sys}_{N}}^{C}(2)-T_{\mathrm{sys}_{N}}^{C}(1)$ becomes directly known from the calibrated NIR measurements during noise injection at both levels. Finally, $v_{o f f}$ is independently estimated using additional detected power levels by means of the variable attenuator inserted in the receiver chain (Fig. 2) [11].

The system temperature at the antenna reference plane is finally computed as

$$
T_{\mathrm{sys}_{k}}=T_{\mathrm{sys}_{k}}^{C} \frac{\left|S_{\mathrm{LC}_{k}}\right|^{2}}{\left|S_{\mathrm{LA}_{k}}\right|^{2} \eta_{k}}
$$

where $S_{\mathrm{LC}_{k}}$ and $S_{\mathrm{LA}_{k}}$ are S parameters of the switch from the $\mathrm{C}$ and antenna ports to the output, respectively, and $\eta_{k}$ is the antenna efficiency. This equation should be applied for both antenna polarization outputs ( $\mathrm{X}$ and $\mathrm{Y}$ ).

\section{Baseline Phase and Amplitude Calibration}

The parameter $G_{k j}$ in (10) is estimated from the measured cross-correlations while injecting the two levels of noise described in Section IV-B. For each level, the correlation temperature $T_{C_{k j}}$ of a given baseline $k j$ at the receivers input plane depends, in general, on the power of the noise source, the distribution network physical temperature and its S-parameters [12]. If two levels are injected, the difference of the corresponding correlation temperatures is directly written in terms of the difference between the system temperatures of the reference receiver (NIR)

$$
T_{C_{k j}}(2)-T_{C_{k j}}(1)=\frac{S_{k 0} S_{j 0}^{*}}{\left|S_{N 0}\right|^{2}}\left[T_{\mathrm{sys}_{N}}^{C}(2)-T_{\mathrm{sys}_{N}}^{C}(1)\right] .
$$

On the other hand, the correlation temperature can be computed by an equation similar to (10)

$$
T_{C_{k j}}(x)=\frac{\sqrt{T_{\mathrm{sys}_{k}}^{C}(x) T_{\mathrm{sys}_{j}}^{C}(x)}}{G_{k j}^{C}} M_{k j}^{C}(x)
$$

where $x=1$ or 2 and $M_{k j}^{c}$ is the quadrature corrected normalized correlation measured while injecting correlated noise. Combining (18) and (19), $G_{k j}^{C}$ can be written in terms of the system temperatures of the receivers, which are computed using (16). The final expression for $G_{k j}^{C}$ shows that it only depends on the quadrature-corrected normalized correlation at both levels of injection, the corresponding PMS voltages, and the relative phases of the NDN S-parameters.

To convert $G_{k j}^{C}$ to the antenna reference plane, the following equation is used:

$$
G_{k j}=G_{k j}^{C} \frac{\bar{S}_{\mathrm{LA}_{k}} \bar{S}_{\mathrm{LA}_{j}}^{*}}{\bar{S}_{\mathrm{LC}_{k}} \bar{S}_{\mathrm{LC}_{j}}^{*}}
$$

where the $S$ parameters are the ones of the switch defined in the previous section and the overbar means normalized to unit amplitude, that is $\bar{S}=S /|S|$. 


\section{Residual Offset}

Thermal noise generated by the common local oscillator and distributed simultaneously to different receivers produces nonzero correlation. This offset is corrected by applying the whole procedure to the data measured while the input switch is in the " $U$ " position, so injecting uncorrelated noise to all receivers. The calibrated visibility measured in this situation is then directly subtracted to the one measured for the switch in antenna positions ("X" or "Y")

$$
V_{k j}=\left.V_{k j}\right|_{\mathrm{X} \text { or } \mathrm{Y} \text { position }}-\left.V_{k j}\right|_{\mathrm{U} \text { position }} \text {. }
$$

\section{Distributed NOISE INJECTION}

Noise injection is accomplished by two kinds of distribution networks. In the central part of the instrument (the hub) there is a 1-to-15 power divider for distributing the noise generated by a single source simultaneously to all hub receivers. As explained in the previous sections, three of them can operate as accurate, highly stable NIR and are used as reference receivers, so the procedure described in the previous section can be directly applied for the hub receivers. On the other hand, each of the three arms is formed by separate sections having six receivers each (Fig. 1) and there is a different noise source at each section. In the present configuration, each source drives a 1-to-12 network for distributing noise to the receivers of the same segment and of the adjacent one. A set of switches allows injecting noise in overlapping sets of receivers to apply the distributed noise injection concept proposed in [13] with the algorithms modified according to the methodology outlined in this paper. In particular, at each segment the reference receiver is chosen as one previously calibrated using another source. The first section of each arm is overlapped with the receivers in the hub that are in line with the arm, so that the noise injection of the first section includes a NIR, which is used to calibrate the rest of receivers in the section. And this process continues for the other sections.

\section{BRIGHTNESS TEMPERATURE IN ANTENNA REFERENCE PlANES}

The brightness temperature map at a given polarization is computed from the calibrated visibility function by inverting the following Fourier-like integral [9]

$$
V_{k j}=\iint_{\xi^{2}+\eta^{2} \leq 1} T_{k j}^{\prime}(\xi, \eta) \overline{\tilde{r}}_{k j}\left(-\frac{u \xi+v \eta}{f_{0}}\right) e^{-j 2 \pi(u \xi+v \eta)} d \xi d \eta
$$

where $\overline{\tilde{r}}_{k j}()$ is the fringe washing function normalized to its value at the origin ( since $\tilde{r}_{k j}(0)$ has been corrected in the calibration process) and $T_{k j}^{\prime}$ the modified brightness temperature

$$
T_{k j}^{\prime}(\xi, \eta)=\frac{\sqrt{D_{k} D_{j}}}{4 \pi} \frac{T_{B}(\xi, \eta)-T_{r}}{\sqrt{1-\xi^{2}-\eta^{2}}} F_{n_{k}}(\xi, \eta) F_{n_{j}}^{*}(\xi, \eta)
$$

where $T_{B}$ is the brightness temperature defined for a field polarization that is selected by the input switch position [14], $T_{r}$ the physical temperature of the receivers, $F_{n}$ the normalized voltage antenna patterns for the corresponding polarization, and $D$ the maximum directivity of the antennas. These ones are assumed at coordinates $\left(x_{k}, y_{k}\right)$ and the following definitions apply: $u=\left(x_{j}-x_{k}\right) / \lambda_{0}$ and $v=\left(y_{j}-y_{k}\right) / \lambda_{0}$ where $\lambda_{0}$ is the wavelength at the center frequency $f_{0}$. Finally, $\xi$ and $\eta$ are the director cosines with respect to the $X$ and $Y$ axes, respectively.

For a given point in the $(u, v)$ plane except the $(0,0)$, the lefthand side of (22) is the calibrated visibility $V_{k j}$ measured by two receivers having relative position given $(u, v)$. In general, for a Y-shape instrument, the redundancy is small and limited only to antennas of the same arm. This means that almost all of the visibility samples are unique. For those samples that are measured simultaneously by different pairs of receivers their average is used, although other approaches are possible. In this way, a function $V(u, v)$ having a unique value for a given pair $(u, v)$ is computed.

The zero baseline $V(0,0)$ is the antenna temperature and is obtained from the calibrated NIR data. Usually radiometers are calibrated using two known loads. However, in the NIR the so-called one-point calibration is applied. This means that the level of the noise injection is determined with one target, after which the antenna temperature can be determined using the physical temperature of the NIR and the ancillary data described in Section II [4]. In orbit, the space background radiation is foreseen to be used as the calibration target for NIR. In consequence the instrument will have to periodically perform a maneuver to point to the cold space.

Since three different NIR are available in the hub, the average of their three calibrated measurements is used as antenna temperature. Furthermore, note that when the NIRs are measuring the fully polarimetric scene brightness temperature, the data can also be used to retrieve visibilities of baselines consisting of the NIR and other receivers. In this case, however, the noise injection mechanism and the Dicke switching of NIR degrade the corresponding visibility samples.

For the third and fourth Stokes parameters the correlations measured between $\mathrm{H}$ - and $\mathrm{V}$-channel need to be calibrated. This can be done as described in Section IV and adding the phase difference of the antenna connecting cables, which is measured on-ground, to the in-phase error.

The first order solution for the inversion of (22) and (23) is an inverse Fourier transform followed by a compensation of the average antenna pattern and the obliquity factor $\sqrt{1-\xi^{2}-\eta^{2}}$. Due to the Y-shape of the instrument, the visibility sampling grid is hexagonal so an hexagonal fast Fourier transform (HFFT) must be used [15]. The whole space maps into the unit circle $\left(\xi^{2}+\eta^{2}<=1\right)$ so $T_{k j}^{\prime}(\xi, \eta)$ is a function bounded by this region. Furthermore, since the antenna separation is larger than the Nyquist sampling spacing, there is some amount of aliasing. Fig. 3 shows the location of the alias of the unit circle and of the earth for a given configuration of platform. In principle, the useful data is the one that is inside the region delimited by the alias of the unit circle. However this one can be extended to the earth alias by appropriate algorithms, as will be explained hereafter.

Improved inversion techniques require the knowledge of $\overline{\tilde{r}}(\tau)$ and of the voltage patterns and maximum directivity of all antennas. The normalized fringe washing function is measured on board during the noise injection mode of operation by correlating the receivers' outputs at three different time delays and approximating the amplitude by a sinc function and the phase 


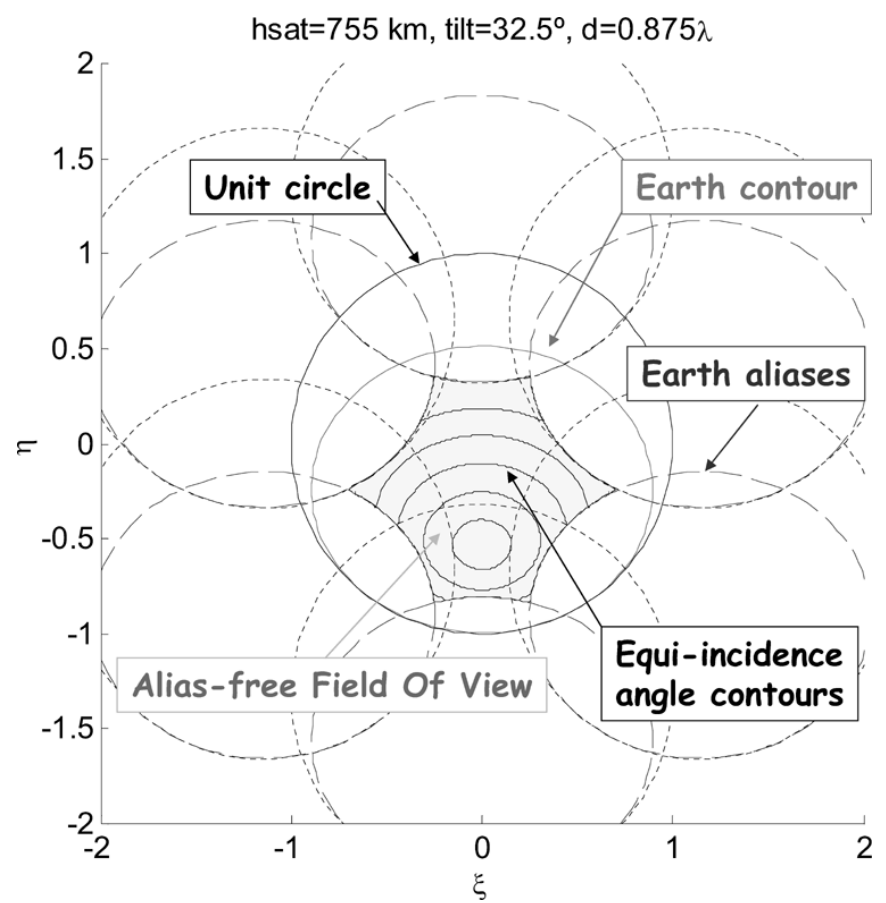

Fig. 3. Field of view of SMOS showing the alias.

by a quadratic polynomial [16]. Due to the distributed noise injection scheme, all receivers are not simultaneously driven by the same noise source and the fringe-washing functions of baselines formed by receivers not sharing a noise source are computed using the approach of [17]. On the other hand, all antenna parameters are assumed to have been measured on ground and that they do not change during the mission lifetime. Measured antenna patterns are used for the inversion of (23).

The proposed image reconstruction algorithm allows to extend the alias-free field of view up to the earth replicas by subtracting to the calibrated visibility function $V(u, v)$ the contributions from 1 ) the physical temperature of the receivers; 2 ) the sky; 3 ) a constant brightness temperature in the region occupied by the earth; and 4) the direct and reflected contributions from the sun and moon which can be determined as in [18]. The differential visibility is then conveniently defined as

$$
\begin{aligned}
\Delta V(u, v)= & V(u, v)-V_{R}-V_{\text {sky }}-T_{\text {Earth }} V_{\text {Earth }} \\
& -T_{\text {Sun,dir }} V_{\text {Sun,dir }}-T_{\text {Sun,ref }} V_{\text {Sun,ref }} \\
& -T_{\text {Moon,dir }} V_{\text {Moon,dir }}-T_{\text {Moon,ref }} V_{\text {Moon,ref }}
\end{aligned}
$$

where the different visibilities are computed by using (22) and (23) with the measured antenna patterns and fringe washing function but substituting $\left(T_{B}(\xi, \eta)-T_{r}\right)$ by the corresponding contribution: the negative of the physical temperature of the receivers $\left(-T_{r}\right)$ for $V_{R}$, the computed brightness temperature of the Sky $\left(T_{B \text { sky }}\right)$ for $V_{\text {sky }}$, a constant 1 for $V_{\text {Earth }}$ and unit amplitude point sources at the directions of the direct and reflected sun and moon for the rest of terms. The value of $T_{\text {Earth }}$ is computed from (24) particularized at the origin $u=v=0$ and by forcing $\Delta V(0,0)=0$.

The inversion algorithm is then applied to the differential visibility, thus obtaining the differential brightness temperature $\Delta T_{B}(\xi, \eta)=T_{B}(\xi, \eta)-T_{\text {Earth }}$ that is, the deviations of the brightness temperature over the mean value in the alias-free field of view. The solution can be reached by discretizing all the visibility samples for all $(\xi, \eta)$ points, and then solving the set of linear equations of the form $\Delta V=G \Delta T_{B}$ where $G$ is the so-called G-matrix, using the following:

- an extension of the CLEAN iterative algorithm, as described in [19];

- pseudoinverse matrix as in [3] in the one-dimensional case;

- conjugate-gradient algorithm as in [20] in the two-dimensional case.

\section{Conversion to Ground ReFEREnCE Frame}

The brightness temperature recovered by visibility inversion is defined at the antenna polarization reference frame. The next step in the level 1 processor consists of applying a rotation matrix to convert the measured brightness temperature to the standard horizontal and vertical frame over the earth's surface.

Due to Faraday rotation effects and to the relative orientation between the pixels reference frame over the earth's surface and the antennas, the electric fields collected $E_{x}, E_{y}$ are rotated an angle $\varphi$ with respect to the field in the h-v frame on the earth's surface $E_{\mathrm{h}}, E_{\mathrm{v}}$

$$
\left[\begin{array}{c}
E_{x} \\
E_{y}
\end{array}\right]=\left[\begin{array}{cc}
\cos \varphi & \sin \varphi \\
-\sin \varphi & \cos \varphi
\end{array}\right]\left[\begin{array}{l}
E_{\mathrm{h}} \\
E_{\mathrm{v}}
\end{array}\right]
$$

Moreover, depending on the input switch positions, three different brightness temperature maps are obtained after image reconstruction: $T_{x x}(\xi, \eta)$ proportional to $\left\langle E_{x} E_{x}^{*}\right\rangle, T_{y x}(\xi, \eta)$ proportional to $\left\langle E_{y} E_{x}^{*}\right\rangle$, and $T_{y y}(\xi, \eta)$ proportional to $\left\langle E_{y} E_{y}^{*}\right\rangle$. The corresponding rotation matrix between them is readily obtained from (25) [14]

$$
\left[\begin{array}{l}
T_{x x} \\
T_{y x} \\
T_{y y}
\end{array}\right]=\left[\begin{array}{cc}
\cos ^{2} \varphi & \sin ^{2} \varphi \\
-\sin 2 \varphi & \sin 2 \varphi \\
\sin ^{2} \varphi & \cos ^{2} \varphi
\end{array}\right]\left[\begin{array}{l}
T_{\mathrm{hh}} \\
T_{\mathrm{vv}}
\end{array}\right]
$$

where $T_{\mathrm{hh}}$ and $T_{\mathrm{vv}}$ are the standard horizontal and vertical brightness temperature of the earth and it has been used the experimental fact that the horizontal and vertical components of the field emitted by the earth at L-band are uncorrelated to each other, resulting that $T_{\mathrm{vh}}$ has negligible values and so it has set equal to 0 .

The pass from the antenna to the earth reference frame consists of inverting (26) in the alias-free field of view pixel to pixel. Note that the antenna reference frame $(x-y)$ is defined by the co-polar and cross-polar directions according to the Ludwig third definition of polarization [21]. This means that the angle $\varphi$ depends on the direction of observation and it is different for each antenna.

MIRAS supports two modes of operation: dual polarization, in which only $T_{x x}(\xi, \eta)$ and $T_{y y}(\xi, \eta)$ are measured, and fullpolarimetric mode where $T_{y x}(\xi, \eta)$ is also measured. For the dual-polarization mode, instead of inverting (26), the following reduced equation must be used:

$$
\left[\begin{array}{l}
T_{x x} \\
T_{y y}
\end{array}\right]=\left[\begin{array}{ll}
\cos ^{2} \varphi & \sin ^{2} \varphi \\
\sin ^{2} \varphi & \cos ^{2} \varphi
\end{array}\right]\left[\begin{array}{l}
T_{\mathrm{hh}} \\
T_{\mathrm{vv}}
\end{array}\right] .
$$

In this case, as already reported previously in [22], the matrix is singular for $\varphi=45^{\circ}$ and some pixels are lost at every snapshot. On the contrary, in the full-polarimetric mode there are no singularities and the whole alias-free field of view can be imaged. Fig. 4 shows a snapshot retrieval for both cases obtained 


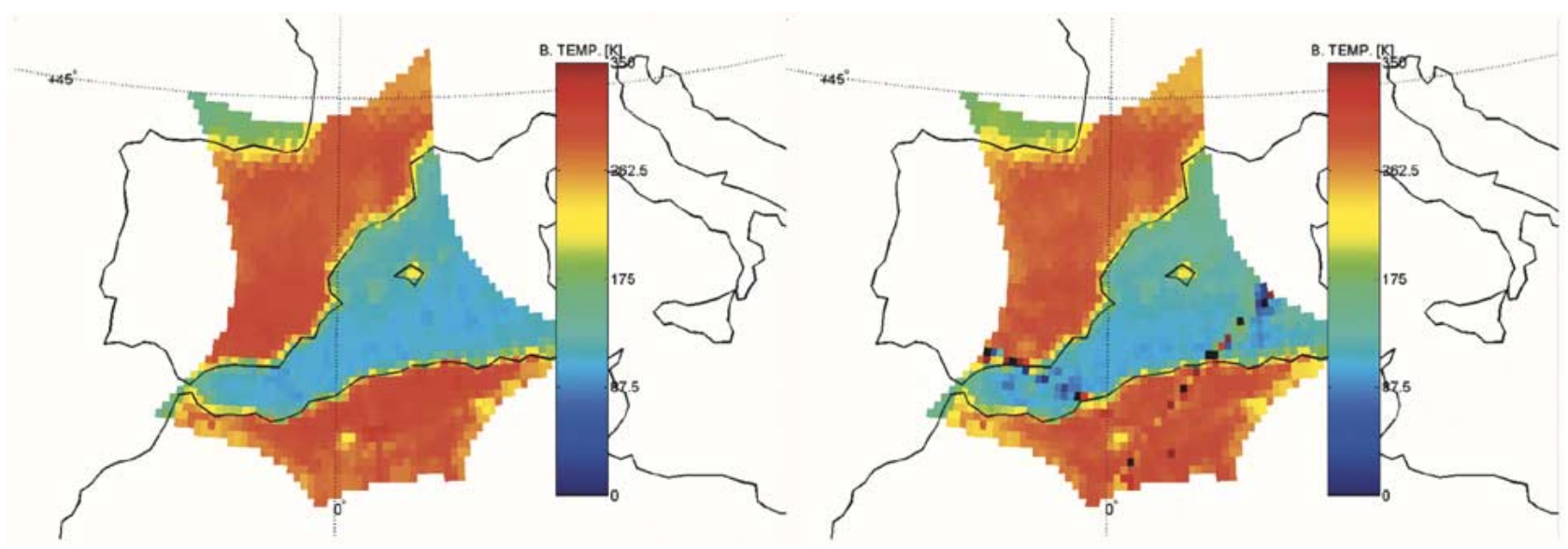

Fig. 4. Images of a single snapshot retrieved with the SMOS End to End Simulator (SEPS) for full-pol (left) and dual-pol (right) modes of operation. Note the singularities at $45^{\circ}$ for the dual-pol mode.

with the SMOS end-to-end performance simulator developed by the authors [23], [24]. The singularities at $\varphi=45^{\circ}$ are clearly seen in the dual-pol case.

On the other hand, in the full-polarimetric mode the integration time per polarization is smaller $(2.4 \mathrm{~s} / 3=0.8 \mathrm{~s})$ than in dual-polarization mode $(2.4 \mathrm{~s} / 2=1.2 \mathrm{~s})$ due to the time sharing between polarization-switch positions. This leads to a radiometric sensitivity increase (i.e., larger noise) by a factor of $\sqrt{3 / 2}=1.22$ for the full-polarimetric case.

Geophysical parameter retrievals in the earth reference frame require a precise knowledge of the observation geometry and of the Faraday rotation, both contributors to the overall rotation angle $\varphi$. However, some parameter retrievals (sea surface salinity and soil moisture over bare or low-vegetated soils) [25] can also be performed in the antenna reference frame, and even working with the first Stokes parameter $I=T_{x x}+T_{y y}=$ $T_{\mathrm{hh}}+T_{\mathrm{vv}}$, which does not require any geometrical or Faraday rotation correction. Faraday rotation using the full-polarimetric mode of MIRAS is studied also in [26].

\section{CONCLUSION}

The specificities of a two-dimensional interferometric aperture synthesis radiometer, such as MIRAS, makes the process of getting the brightness temperature map on the earth's surface from the instrumental raw data very complex. In particular, the data processing must strongly rely on the hardware solutions chosen to correct for non ideal behavior of the electronic components. Also a complete understanding of the theory behind the interferometric aperture synthesis technique, including the simplifications and approximations carried out, is essential to achieve accurate image reconstruction algorithms, especially due to the aliasing condition produced by the sparse sampling in the $(\mathrm{u}, \mathrm{v})$ domain and the important role that the antenna patterns and fringe washing functions play. Polarization issues must also be taken into account due to the large field of view of the instrument.

All processing algorithms are being tested using the end-toend simulator developed by the authors in the frame of the SMOS project and the results show that the spaceborne instrument will be able to produce brightness temperature maps with the required accuracy and resolution both from dual-pol or full-polarimetric measurements.
The operating mode of MIRAS will be in the end dependent on the type of scene being imaged: over the ocean for example, simulation and experimental results show that the sea surface salinity can be retrieved effectively by using the first Stokes parameter and this can be obtained with only the dual-polarization mode. On the other hand, soil moisture over high vegetated soils may require the full-polarimetric data.

\section{REFERENCES}

[1] P. Silvestrin, M. Berger, Y. Kerr, and J. Font, "ESA's second Earth Explorer Opportunity Mission: The Soil Moisture and Ocean Salinity mission-SMOS," IEEE Geosci. Remote Sens. Newslett., no. 118, pp. 11-14, Mar. 2001.

[2] M. Martín-Neira and J. M. Goutoule, "A two-dimensional aperture-synthesis radiometer for soil moisture and ocean salinity observations," ESA Bull., no. 92, pp. 95-104, Nov. 1997.

[3] C. S. Ruf, C. T. Swift, A. B. Tanner, and D. M. Le Vine, "Interferometric synthetic aperture microwave radiometry for the remote sensing of the Earth," IEEE Trans. Geosci. Remote Sens., vol. 26, no. 5, pp. 597-611, Sep. 1988.

[4] A. Colliander, S. Tauriainen, T. Auer, J. Kainulainen, J. Uusitalo, M. Toikka, and M. Hallikainen, "Calibration of fully polarimetric noise injection radiometer of MIRAS," in 8th Specialist Meeting on Microwave Radiometry and Remote Sensing Applications - $\mu$ Rad 2004, Univ. La Sapienza, Rome, Italy, Feb. 24-27, 2004, p. 80.

[5] F. T. Ulaby, R. K. Moore, and A. K. Fung, Microwave Remote Sensing. Active and Passive. Norwood, MA: Artech House, 1981, vol. 1, Fundamentals and Radiometry.

[6] J. B. Hagen and D. T. Farley, "Digital-correlation techniques in radio science," Radio Sci., vol. 8, no. 8,9, pp. 775-784, Aug.-Sep. 1973.

[7] M. Martín-Neira, S. Ribó, and K. Rautiainen, "0-1 correction of comparator threshold in 1-bit interferometric radiometers," in 8th Specialist Meeting on Microwave Radiometry and Remote Sensing Applications $-\mu$ Rad 2004, Univ. La Sapienza, Rome, Italy, Feb. 24-27, 2004, p. 93.

[8] I. Corbella, F. Torres, A. Camps, and J. Bará, "A new calibration technique for interferometric radiometers," in Proc. Eur. Symp. Aerospace Remote Sensing. Conf. Sensors, Systems and Next Generation Satellites IV, Barcelona, Spain, Sep. 1998, pp. 359-366.

[9] I. Corbella, N. Duffo, M. Vall-llossera, A. Camps, and F. Torres, "The visibility function in interferometric aperture synthesis radiometry," IEEE Trans. Geosci. Remote Sens., vol. 42, no. 8, pp. 1677-1682, Aug. 2004.

[10] F. Torres, N. Duffo, I. Corbella, A. Camps, and M. Vall-llossera, "MIRAS-SMOS, the relative instrumental error correction approach," in 8th Specialist Meeting on Microwave Radiometry and Remote Sensing Applications $-\mu \operatorname{Rad} 2004$, Univ. La Sapienza, Rome, Italy, Feb. 24-27, 2004, p. 84.

[11] F. Torres, N. Duffo, I. Corbella, A. Camps, M. Vall-llossera, and L. Sagués, "Dynamic range and linearity tradeoff in detectors for interferometric radiometers," IEE Electron. Lett., vol. 39, no. 25, pp. 1852-1854, Dec. 11th, 2003. 
[12] I. Corbella, A. Camps, F. Torres, and J. Bará, "Analysis of noise injection networks for interferometric radiometer calibration," IEEE Trans. Microw. Theory Tech., vol. 48, no. 4, pp. 545-552, Apr. 2000.

[13] F. Torres, A. Camps, J. Bará, I. Corbella, and R. Ferrero, "On-board phase and modulus calibration of large aperture synthesis radiometers: Study applied to MIRAS," IEEE Trans. Geosci. Remote Sens., vol. 34, no. 4, pp. 1000-1009, Jul. 1996.

[14] M. Martín-Neira, S. Ribó, and A. J. Martín-Polegre, "Polarimetric mode of MIRAS," IEEE Trans. Geosci. Remote Sens., vol. 40, no. 8, pp. 1755-1768, Aug. 2002.

[15] A. Camps, J. Bará, I. Corbella, and F. Torres, “The processing of hexagonally sampled signals with standard rectangular techniques: application to 2D large aperture synthesis interferometric radiometers," IEEE Trans. Geosci. Remote Sens., vol. 35, no. 1, pp. 183-190, Jan. 1997.

[16] A. Camps, F. Torres, I. Corbella, J. Bará, and F. Monzón, "Automatic calibration of channels frequency response in interferometric radiometers," Electron. Lett., vol. 35, no. 1, pp. 115-116, Jan. 1999.

[17] R. Butora, M. Martín-Neira, and A. L. Rivada-Antich, "Fringe-washing function calibration in aperture synthesis microwave radiometry," Radio Sci., vol. 38, no. 2, pp. 15-1-15-5, 2003. DOI: 10.1029/2002RS002695.

[18] A. Camps, M. Vall-llossera, N. Duffo, M. Zapata, I. Corbella, F. Torres, and V. Barrena, "Sun effects in 2-D aperture synthesis radiometry imaging and their cancellation," IEEE Trans. Geosci. Remote Sens., vol. 42, no. 6, pp. 1161-1167, Jun. 2004

[19] A. Camps, J. Bará, F. Torres, and I. Corbella, "Extension of the CLEAN technique to the microwave imaging of continuous thermal sources by means of aperture synthesis radiometers," Progr. Electromagn. Res., vol. 18, pp. 67-83, Jan. 1998

[20] R. Tomás, "Algoritmos de reconstrucción de imagen en radiometría por síntesis de apertura," School of Telecommun, Eng., Univ. Politècnica de Catalunya, Barcelona, Spain, Final Project Report, 2002.

[21] A. C. Ludwig, "The definition of cross polarization," IEEE Trans. Antennas Propagat., vol. AP-21, no. 1, pp. 116-119, Jan. 1973.

[22] P. Waldteufel and G. Caudal, "About off-axis radiometric polarimetric measurements," IEEE Trans. Geosci. Remote Sens., vol. 40, no. 6, pp. 1435-1439, Jun. 2002.

[23] I. Corbella, A. Camps, M. Zapata, F. Marcos, F. Martínez, F. Torres, M. Vall-llossera, N. Duffo, and J. Bará, "End to end simulator of 2D interferometric radiometry," Radio Sci., vol. 38, no. 3, 8058, pp. 23-1-23-7, Jun. 2003. DOI: 10.1029/2002RS002665.

[24] A. Camps, I. Corbella, M. Vall-llossera, N. Duffo, F. Marcos, F. Martínez-Fadrique, and M. Greiner, "The SMOS end-to-end performance simulator: Description and scientific applications," in Proc. IGARSS, vol. 1, Toulouse, France, Jul. 21-25, 2003, pp. 13-15.

[25] A. Camps, I. Corbella, M. Vall-llossera, R. Villarino, L. Enrique, F. Julbé, J. Font, A. Juliá, C. Gabarró, J. Etchetto, J. Boutin, A. Weill, F. Torres, N. Duffo, E. Rubio, V. Caselles, P. Wursteisen, and M. Martín-Neira, "L-band sea surface emissivity: preliminary results of the WISE-2000 campaign and its application to salinity retrieval in the SMOS mission," Radio Sci., vol. 38, no. 4-8071, Jun. 19, 2003. DOI: $10.1029 / 2002$ RSO02629.

[26] S. Ribó and M. Martín-Neira, "Faraday rotation correction in the polarimetric mode of MIRAS," IEEE Trans. Geosci. Remote Sens., vol. 42, no. 7, pp. 1405-1410, Jul. 2004.

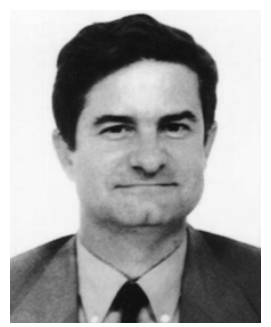

Ignasi Corbella (M'99) received the Telecommunications Engineering and Dr.Eng. degrees, both from Universitat Politècnica de Catalunya (UPC), Barcelona, Spain, in 1977 and 1983, respectively.

In 1976, he joined the School of Telecommunication Engineering in UPC as a Research Assistant in the Microwave Laboratory, where he worked on passive microwave integrated circuit design and characterization. During 1979, he worked at ThomsonCSF, Paris, France, on microwave oscillators design. In 1982, he became an Assistant Professor at UPC, an Associate Professor in 1986, and a Full Professor in 1993. He is currently teaching microwaves at the undergraduate level in UPC and has designed and taught graduate courses on nonlinear microwave circuits. During the school year 1998-1999, he worked at NOAA/Environmental Technology Laboratory, Boulder, CO, as a Guest Researcher, developing methods for radiometer calibration and data analysis. His research work in the Department of Signal Theory and Communications, UPC includes microwave airborne and satellite radiometry and microwave system design.

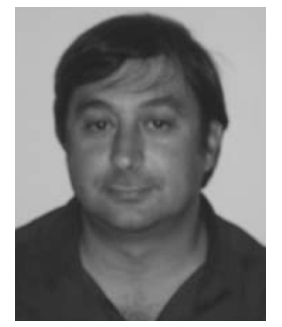

Francesc Torres (M'96) received the Ingeniero and Doctor Ingeniero degrees in telecommunication engineering from the Polytechnic University of Catalonia (UPC), Barcelona, Spain, in 1988 and 1992 , respectively.

In 1988-1989, he was a Research Assistant in the RF System Division, European Space Agency, Noordwijk, The Netherlands, devoted to microwave device testing and characterization. In 1989, he joined the Antenna-Microwave-Radar group, UPC, where he is currently an Associate Professor. His main research interests are focused on the design and testing of microwave systems and subsystems. He is currently engaged in research on interferometric radiometers devoted to earth observation.

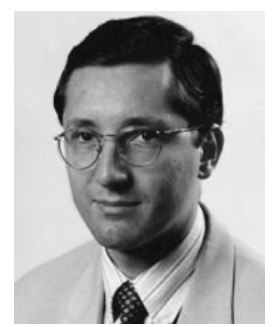

Adriano Camps (S'91-A'97-M'00-SM'02) was born in Barcelona, Spain, in 1969. He received the Telecommunications Engineering degree and the $\mathrm{Ph} . \mathrm{D}$. degree in telecommunications engineering in 1992 and 1996, respectively, both from the Polytechnic University of Catalonia (UPC), Barcelona, Spain

From 1991 to 1992, he was with the ENS des Télécommunications de Bretagne, Bretagne, France, with an Erasmus Fellowship. In 1993, he joined the Electromagnetics and Photonics Engineering group, at the Department of Signal Theory and Communications, UPC, as an Assistant Professor, and since 1997 as an Associate Professor. In 1999, he was on sabbatical leave at the Microwave Remote Sensing Laboratory, University of Massachusetts, Amherst. His research interests are microwave remote sensing, with special emphasis in microwave radiometry by aperture synthesis techniques. He has performed numerous studies within the frame of European Space Agency SMOS Earth Explorer Mission. He is an Associate Editor of Radio Science.

Dr. Camps received the second national award of university studies in 1993, the INDRA award of the Spanish Association of Telecommunication Engineering to the best Ph.D. in 1997, the extraordinary Ph.D. award at the Universitat Politècnica de Catalunya in 1999, the First Duran Farell Award and the Ciudad de Barcelona Award, in 2000 and 2001, respectively, both for Technology Transfer, in 2002, the Research Distinction of the Generalitat de Catalunya for contributions to microwave passive remote sensing, in 2003 , the Premi Nacional de Telecomunicacions (Generalitat de Catalunya) with the members of the Electromagnetics and Photonics Engineering group, and in 2004 a EURYI (European Young Investigator) Award. Also, as a member of the Microwave Radiometry Group at UPC, he received in 2000, 2001, and 2004 the 1st Duran Farell and the Ciudad de Barcelona Awards for Technology Transfeer, and the Salvà i Campillo Award of the Telecommunications (Engineering College of Catalonia) to the most innovative research project. He was Chair of $\mathrm{Cal}$ '01. He is editor of the IEEE Geoscience and Remote Sensing Newsletter and President-Founder of the IEEE Geoscience and Remote Sensing Society Spain Chapter

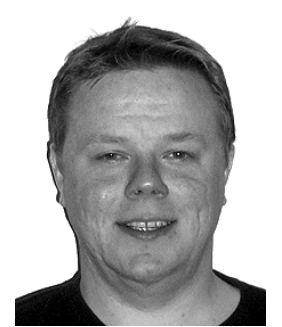

Andreas Colliander was born in 1976 in Imatra, Finland. He received the M.Sc. degree from the Helsinki University of Technology (TKK), Espoo, Finland, in 2002.

$\mathrm{He}$ is currently a Research Scientist and Project Manager in the Laboratory of Space Technology of TKK, and pursuing his studies toward the D.Sc. degree. He has a research student position in the national Graduate School for Remote Sensing, which is supported by Finnish Ministry of Education and Academy of Finland. His research is focused on microwave radiometer systems, with emphasis on polarimetric and interferometric radiometers, and on theoretical simulation of rough surface backscattering. $\mathrm{He}$ has authored and coauthored 11 scientific publications on microwave remote sensing.

Mr. Colliander received the 2002 TKK Master's Thesis Award, an annual award for top five Master's thesis of TKK. 


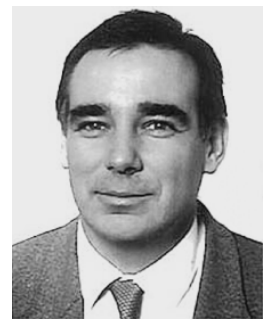

Manuel Martín-Neira (M'96) received the M.S. and $\mathrm{Ph} . \mathrm{D}$. degrees in telecommunication engineering in 1986 and 1996, respectively, from the School of Telecommunication Engineering, Polytechnical University of Catalonia, Catalonia, Spain.

From 1989 to 1992, he was was with GMV, a Spanish firm, where he was responsible for several projects for the European Space Agency (ESA) related to global positioning satellite navigation with applications to precise landing and attitude determination. Since 1992, with ESA, he has been in charge of the radiometer activities within the Payload, Equipment, and Technology Section. During this period, he has been responsible for the predevelopment technology activities related to the Microwave Imaging Radiometer with Aperture Synthesis (MIRAS) project. MIRAS (L-band Microwave Imaging Radiometer with Aperture Synthesis) is the only payload onboard the Soil Moisture and Ocean Salinity mission (SMOS), ESA's second Earth Explorer Opportunity Mission. He is currently the SMOS Instrument Principal Engineer.

Dr. Martín-Neira was awarded a Fellowship to work on radiometry at European Space Research and Technology Center (ESTEC), Noordwijk, The Netherlands, in 1988.

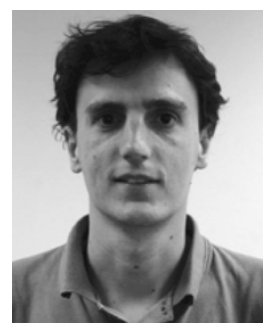

Serni Ribó received the M.S. degree in telecommunication engineering from the Universitat Politècnica de Catalunya, Catalonia, Spain, in 1999. He is currently pursuing the Ph.D. degree from the Universitat Politècnica de Catalunya.

He was with Sony España as Electrical Design Engineer for rear projector televisions. Later in 2000, he joined the European Space Agency, with a grant from the Spanish Ministry for Science and Technology, were he worked with synthetic aperture radiometry technology aspects. In 2002 , he joined the Laboratory of Space Technology, Helsinki University of Technology during three months were he collaborated in the development of the two-dimensional synthetic aperture radiometer HUT-2 D. Since 2003, he has been with the Space Research Institute of Catalonia, where he is continuing research on synthetic aperture radiometry applied to the SMOS mission of the European Space Agency.
Kimmo Rautiainen was born 1969 in Säkylä, Finland. He received the M.Sc. degree from the Helsinki University of Technology (TKK), Espoo, Finland, in 1996.

He is currently a Research Scientist and Project Manager in the Laboratory of Space Technology, TKK. His research is focused on microwave radiometer systems, with emphasis on interferometric radiometers. He is currently responsible of technical matters on an ariborne two-dimensional interferometric radiometer.

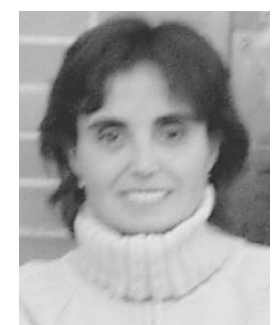

Núria Duffo (S'91-M'99) received the Telecommunication Engineer degree from the Polytechnic University of Catalonia (UPC), Barcelona, Spain, and the Doctor in Telecommunication Engineering from UPC, in 1990 and 1996, respectively.

Since 1997, she has been an Associate Professor at UPC. Her current research interests are numerical methods in electromagnetics, microwave radiometry, antenna analysis, and design.

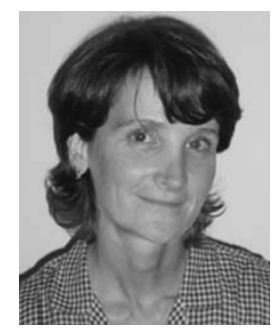

Mercè Vall-Ilossera (M'99) received the Senior Telecommunication Engineer and the Doctor Telecommunication Engineering degrees in 1990 and 1994, respectively, both from the Polytechnic University of Catalonia (UPC), Barcelona, Spain.

She has been lecturing and doing research at the Department of Signal Theory and Communications, UPC from 1990 until 1997 as an Assistant Professor and from 1997 until present as an Associate Professor. She spent a sabbatical year in Montreal with the scholarship of the "Programme Québécois de Bourses d'excellence" (1996-1997): "Stages de Formation postdoctorale au Québec pour jeunes diplômés étrangers." Her research interests include numerical methods in electromagnetism, microwave radiometry, antenna analysis, and design. Currently, her research is mainly related to the study of numerical methods applied to the sea surface emissivity and their characterization at L-band and the MIRAS/SMOS project.

Dr. Vall-llossera, along with the other members of the radiometry group at UPC, was awarded the "9th Edition of the Salvà i Campillo Award" in 2004, the "Primer Premio Duran Farell de Investigación Tecnológica" in 2002, and the "Primer Premio Ciutat de Barcelona d'Investigació Tecnològica" in 2001. 